\title{
Severe Pneumonia Caused by Streptococcus Pneumoniae and Haemophilus Influenzae Coinfection in an Elderly Patient: A Case Report
}

\author{
Nobuhiro Akuzawaa, c, Naoyuki Harada ${ }^{\mathrm{a}}$, Noriko Hasegawa ${ }^{\mathrm{a}}$, Hidenori Seki ${ }^{\mathrm{a}}$, Yuko Oku ${ }^{\mathrm{a}}$, \\ Masayuki Totsuka ${ }^{a}$, Takashi Hatori ${ }^{a}$, Jun Murakami ${ }^{a}$, Kunihiko Imai ${ }^{a}$, \\ Yonosuke Kitahara ${ }^{a}$, Masahiko Tashiro ${ }^{a}$, Masahiko Kurabayashi ${ }^{b}$
}

\begin{abstract}
An 80-year-old man was admitted to our hospital because of worsening dyspnea, fever and sputum. Both chest X-ray film and computed tomography findings showed localized consolidation of the right lower lung. A urine sample was positive for Streptococcus pneumoniae antigen. After initial treatment with meropenem for 3 days, his symptoms improved and high-dose ampicillin was substituted for meropenem. However, 3 days later he required intubation for adult respiratory distress syndrome. We reinstituted meropenem therapy and this proved successful. Sputum culture revealed coinfection with penicillin sensitive $S$. pneumoniae and $\beta$-lactamasenegative ampicillin-resistant Haemophilus influenzae.
\end{abstract}

Keywords: Streptococcus pneumoniae; $\beta$-lactamase-negative ampicillin-resistant Haemophilus influenzae; Adult respiratory distress syndrome

\section{Introduction}

Even nowadays, community-acquired pneumonia (CAP) continues to be one of the commonest causes of morbidity and mortality worldwide, particularly in the elderly. Epidemiologic studies show that pneumonia ranks fourth among leading causes of death in Japan. Streptococcus pneumoniae

\footnotetext{
Manuscript accepted for publication July 27, 2012

${ }^{a}$ Department of Internal Medicine, Social Insurance Gunma Chuo General Hospital, 1-7-13, Koun-cho, Maebashi, Gunma 371-0025, Japan

${ }^{\mathrm{b}}$ Department of Medicine and Biological Science, Gunma University Graduate School of Medicine, 3-39-22, Showa-machi, Maebashi, Gunma 371-8511, Japan

${ }^{\mathrm{c}}$ Corresponding author: Nobuhiro Akuzawa, Department of Internal Medicine, Social Insurance Gunma Chuo General Hospital, 1-7-13, Koun-cho, Maebashi, Gunma 371-0025, Japan.

Email: akuzawa_nobuhiro@yahoo.co.jp

doi: http://dx.doi.org/10.4021/jmc843w
}

is an important pathogen in many community-acquired respiratory infections, including CAP, acute bacterial sinusitis, acute otitis media, and in more invasive infections such as meningitis and bacteremia.

The $\beta$-Lactam antibiotics have been very effective against $S$. pneumoniae; however, resistance to this class of antibiotic has become an increasing problem. Haemophilus influenzae is also a common pathogen in patients with adult CAP and pediatric otitis media and bacterial meningitis. The prevalence of ampicillin resistant strains among $H$. influen$z a e$ isolated from the lower respiratory tract of Japanese children has recently increased significantly $[1,2]$. H. influenzae type $\mathrm{b}$ (Hib) is believed to be responsible for serious illness, chiefly meningitis and pneumonia, worldwide. In particular, meningitis remains a serious problem in children in areas where Hib vaccine is not used. In Japan, meropenem plus an expanded-spectrum cephalosporin such as cefotaxime is recommended for treatment of $H$. influenzae meningitis [3].

Here, we describe a patient who we initially diagnosed as having bacterial pneumonia caused by $S$. pneumoniae infection. His illness progressed to adult respiratory distress syndrome a few days after we substituted high-dose ampicillin for meropenem. We found that this progression was due to coinfection with $\beta$-lactamase-negative ampicillin-resistant (BLNAR) H. influenzae.

\section{Case Report}

An 80-year-old man was admitted to the emergency ward of our hospital in May 2010 because of dyspnea, brown sputum and chills due to a high fever that he had had for 2 days. He had developed a common cold $1 \mathrm{wk}$ before this admission. He had chronic gastritis and hypertension that was being treated with amlodipine, valsartan and famotidine. He had no history of any respiratory disease and smoking.

On admission, physical examination revealed a high body temperature $\left(39.2^{\circ} \mathrm{C}\right)$ and moist rales in the right lower chest. Shortness of breath was obvious; his blood oxygen saturation $\left(\mathrm{SpO}_{2}\right)$ was $92 \%$ on room air. Laboratory findings indicated mild inflammation as follows: WBC count, $12,000 / \mathrm{mm}^{3}$; neutrophils, $85.0 \%$; and C-reactive protein 

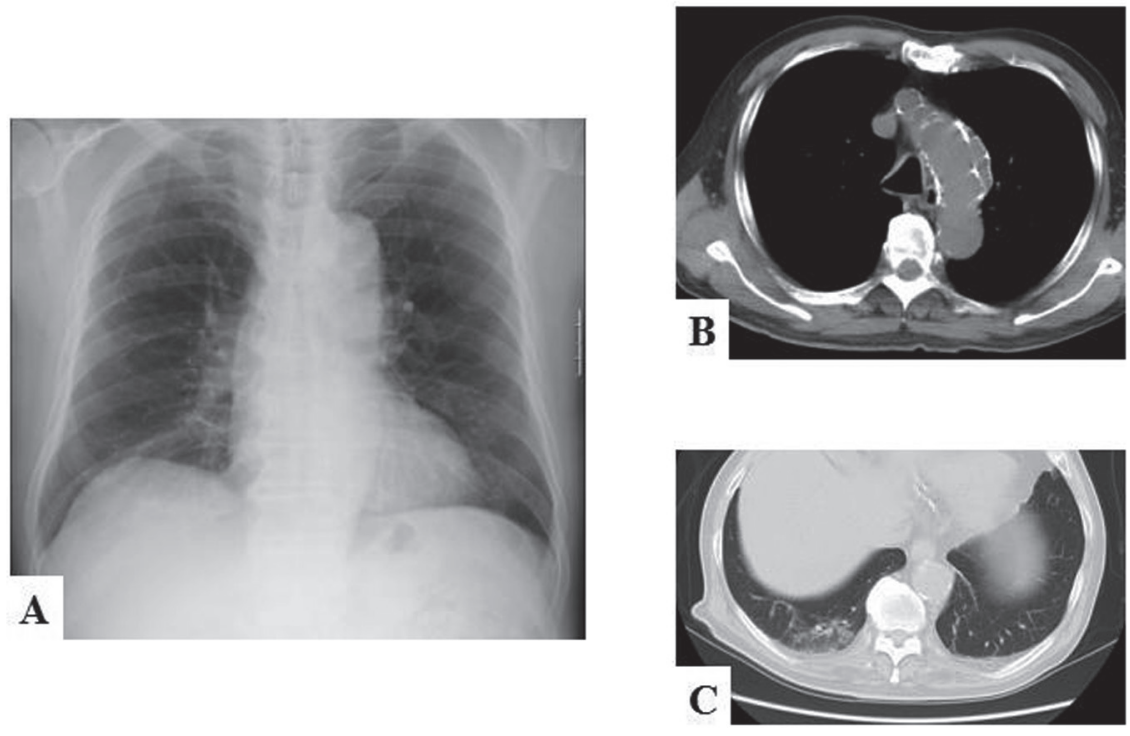

Figure 1. (A) Chest X-ray film and (B, C) computed tomograms of the patient on admission. (A) Mild consolidation is apparent along the right lower lobe branch of the pulmonary artery. The silhouette sign is negative, suggesting the right lower lobe is the site of the pneumonia. (B) Computed tomogram shows an old dissecting aneurysm of the aortic arch (white arrows). (C) The pneumonia mainly affects S10 of the right lower lobe.

(CRP), $2.84 \mathrm{mg} / \mathrm{dL}$. His fasting plasma glucose concentration was $160 \mathrm{mg} / \mathrm{dL}$ and $\mathrm{HbAlc} 7.2 \%$, suggesting untreated diabetes mellitus. Other data were normal. We found limited consolidation of the right lower lung on radiographs. Further investigation by computed tomography revealed alveolar infiltration in the right lower lobe and an old dissecting aneurysm of the aortic arch (Fig. 1). However, he had not experienced severe chest pain. Gram stained sputum smear samples revealed a number of Gram-positive diplococci and some phagocytosis. A urine sample was positive for S. pneumoniae antigen. A nasopharyngeal swab was negative for influenza virus type-A and B antigens and his urine was negative for Legionella pneumophila antigen. Based on these findings, we considered that $S$. pneumoniae was the most likely cause of his bacterial pneumonia. However, we could not exclude the possibility that he was infected with penicillin-intermediate $S$. pneumoniae (PISP) or penicillinresistant $S$. pneumoniae (PRSP). We began intravenous administration of meropenem $(1.5 \mathrm{~g} /$ day $)$ immediately after hospitalization.

By day 3, his laboratory data had improved: his WBC count was $9,700 / \mathrm{mm}^{3}$ and CRP concentration $3.50 \mathrm{mg} / \mathrm{dL}$. His body temperature was normal and his appetite better. His symptoms of moist cough, headache and general fatigue had also dramatically improved. His partial pressure of $\mathrm{O}_{2}$ $\left(\mathrm{PaO}_{2}\right)$ was 89.3 Torr while inhaling $1 \mathrm{~L} /$ min oxygen via a nasal cannula. Because high-dose penicillin can also be effective in the treatment of $S$. pneumoniae infection, including PISP and PRSP, we reconsidered whether meropenem was the most appropriate antibiotic for him. We consulted our hospital's Infection Control Team and decided to accept their recommendation to substitute ampicillin $(8 \mathrm{~g} /$ day $)$ for the meropenem.

He began high-dose ampicillin on day 4. By that evening, his body temperature had increased to $37.8{ }^{\circ} \mathrm{C}$ and he was complaining of chills. On the morning of day 5 , his $\mathrm{SpO}_{2}$ suddenly decreased to $87 \%$ and his body temperature increased to $39.4^{\circ} \mathrm{C}$. He was experiencing difficulty in breathing in spite of inhaling $10 \mathrm{~L} / \mathrm{min}$ oxygen via a face mask. Blood gas analysis revealed hypoxemia $\left(\mathrm{PaO}_{2} 65.3\right.$ Torr) and hypocapnia (partial pressure of $\mathrm{CO}_{2}\left(\mathrm{PaCo}_{2}\right) 35.2$ Torr), indicating he was compensating for respiratory failure by hyperventilating. His WBC count had increased to $12,500 / \mathrm{mm}^{3}$ and his CRP concentration to $15.83 \mathrm{mg} / \mathrm{dL}$. His chest X-ray films had worsened, showing bilateral broad infiltration of the lungs (Fig. 2). An echocardiographic study performed at the patient's bedside showed a normal left ventricular contraction and relaxation pattern. We therefore concluded that the pulmonary edema was non-cardiogenic and that he had acute adult respiratory distress syndrome due to bacterial pneumonia.

On the same day, penicillin-sensitive $S$. pneumoniae (PSSP) and BLNAR H. influenzae were detected in the sputum that had been collected at the time of hospital admission. BLNAR $H$. influenzae was sensitive to cefotaxime, ceftazidime, ceftriaxone, clarithlomycin, levofloxacin, ciprofloxacin and meropenem. Because PSSP is sensitive to ampicillin, we interpreted these results as indicating that BLNAR $H$. influenzae had caused the worsening of his pneumonia after the change in his antibiotic regime. We therefore resumed 

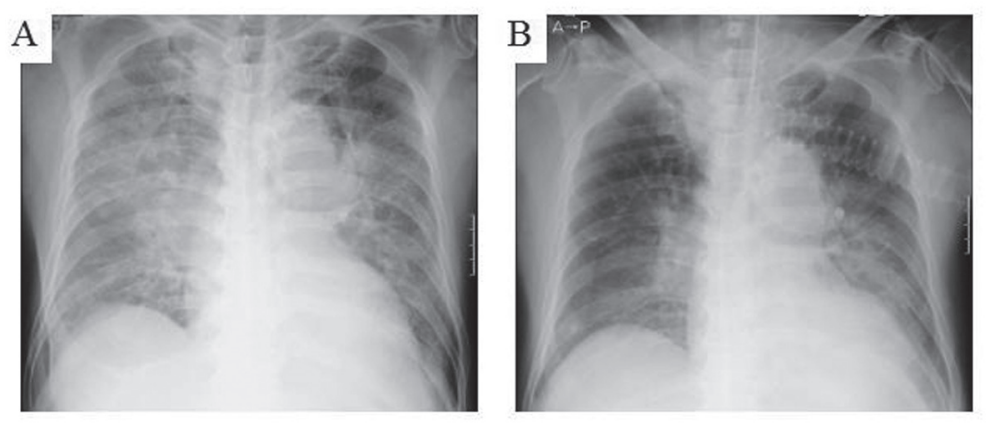

Figure 2. Chest X-ray films on (A) day 5 and $(B)$ day 10. (A) Bilateral infiltration of the lung, which was not present on day 3 , is now apparent. (B) The radiographic findings have improved considerably by 4 days after intubation (day 10). Although some infiltration remains, the $\mathrm{SpO}_{2}$ is now $100 \%$ on $30 \% \mathrm{FiO}_{2}$, accordingly we extubated him.

administration of meropenem (2.0 g/day) and discontinued the high-dose ampicillin therapy.

Although his condition was worse on day 6, we continued to administer meropenem, which is effective against both PSSP and BLNAR $H$. influenzae infection. He had extreme tachypnea of 40 breaths/min with difficulty in expectoration. His $\mathrm{SpO}_{2}$ had decreased to $78 \%$ on $10 \mathrm{~L} / \mathrm{min}$ $\mathrm{O}_{2}$ by face mask. We decided to prescribe sivelestat sodium hydrate, a potent inhibitor of neutrophil-derived elastase and placed him on a ventilator. He required $100 \%$ fraction of inspired $\mathrm{O}_{2}\left(\mathrm{FiO}_{2}\right)$ to maintain his $\mathrm{SpO}_{2}$ above $90 \%$.

His respiratory status began to improve the next day (day 7). His $\mathrm{SpO}_{2}$ was $95 \%$ on $50 \% \mathrm{FiO}_{2}$ and his laboratory data still showed evidence of severe inflammation with WBC count $8,100 / \mathrm{mm}^{3}$ and CRP concentration of $21.77 \mathrm{mg} /$ dL. We consulted the Nutrition Support Team and accepted their recommendation to introduce enteral nutrition with $\mathrm{Ox}$ epa (Abott, Tokyo, Japan), which has been clinically shown to modulate the inflammatory response in critically ill, mechanically ventilated patients, especially those with systemic inflammatory response syndrome, acute lung injury or acute respiratory syndrome. We also asked the physical therapists to institute a rehabilitation program to prevent muscle weakening and contracture, and they immediately began passive exercises.

The patient's $\mathrm{SpO}_{2}$ had recovered to $98 \%$ on $40 \% \mathrm{FiO}_{2}$ on day 8 and his body temperature had dropped to normal by day 9. His $\mathrm{SpO}_{2}$ was stable at $100 \%$ on $30 \% \mathrm{FiO}_{2}$. We confirmed that he could breathe spontaneously without mechanical ventilation support and successfully withdrew him from artificial respiration on day 10 . Fortunately, he showed no neurological deficits from hypoxia-induced brain damage.

His clinical course after extubation was excellent. He could take water and liquid food orally by day 12 , when we removed his feeding tube. By day 13 his laboratory data had improved dramatically with WBC count $7,800 / \mathrm{mm}^{3}$ and CRP concentration decreased to $0.32 \mathrm{mg} / \mathrm{dL}$. We therefore stopped administering meropenem and he continued rehabilitation for about $3 \mathrm{wks}$, including leg muscle strengthening exercises. We also prescribed a diabetic diet. We discharged him from our hospital free of any physical problems on day 30 .

\section{Discussion}

S. pneumoniae has been recognized as the commonest cause of CAP $[4,5]$. Miyashita et al reported the incidence of causative pathogens in ambulatory and hospitalized patients with CAP in Japan [6]. According to their report, Mycoplasma pneumoniae is the commonest pathogen found in such outpatients and $S$. pneumoniae the commonest in hospitalized patients. They drew further distinctions between the pathogens found in outpatients and hospitalized patients with CAP, namely that the incidence of infection with $M$. pneumoniae and is higher among outpatients than among hospitalized patients, whereas the incidence of infection with $S$. pneumoniae and $H$. influenzae is higher among hospitalized patients. They concluded that $S$. pneumoniae and the atypical pathogens, C. pneumoniae and $M$. pneumoniae, are significant causative micro-organisms in both outpatients and hospitalized patients with CAP in Japan.

The $\beta$-Lactam antibiotics are very effective against $S$. pneumoniae; however, resistance to this class of antibiotics has become an increasing problem. PRSP became widespread in the 1980s [7]. Genetic analysis has revealed that PRSP acquire resistance to penicillin by extensive remodeling of the transpeptidase domain of penicillin-binding protein (PBP) 2B; this reduces their affinity for $\beta$-lactams [8]. Therefore, high-dose penicillin is indispensable to the treatment of pneumonia caused by PRSP, and the customary doses of compound antibiotics with $\beta$-lactamase inhibitors such as sulbactam/ampicillin and clavulanic acid/amoxicillin may not produce the desired effects. A retrospective study of 306 Japanese patients with pneumonia caused by $S$. pneumoniae showed that use of $\beta$-lactams in the previous 3 months and the presence of chronic obstructive pulmonary disease were associated with penicillin resistance. The failure rate of first 
line antibiotics was significantly higher in the resistant group $(22.5 \%)$ than in the sensitive group $(9.0 \%)$ [9]. They also reported that penicillin sensitive and resistant organisms were responsible for $177(57.7 \%)$ and $129(42.3 \%)$ cases of pneumonia, respectively.

On the other hand, with $H$. influenzae, resistance to ampicillin and other $\beta$-lactam antibiotics is generally limited to either production of a $\beta$-lactamase or, in the case of BLNAR strains, the presence of altered PBPs with reduced affinity for $\beta$-lactams [10]. A very small proportion of strains possesses both mechanisms; these are referred to as $\beta$-lactamasepositive amoxicillin-clavulanate-resistant strains [11]. Although S. pneumoniae and $M$. pneumoniae are the commonest pathogens in adult CAP patients, $H$. influenzae causes 13\% of cases in Japan [6]. The American Thoracic Society and the Infectious Disease Society of America have established treatment guidelines for management of CAP in immunocompetent adults [12, 13]. Recommendations for inpatients in medical wards include levofloxacin, gatifloxacin, gemifloxacin, or moxifloxacin alone or azithromycin or clarithromycin plus ceftriaxone, ampicillin-sulbactam, and ertapenem.

In Japan, the Japanese Respiratory Society (JRS) has provided guidelines for the management of CAP in adults, including an update and new recommendations in 2006 [14]. To assess CAP severity, the guideline committee advocates a simplified scoring system based on characteristics in Japan and including the following five variables: age (men $\geq$ $70 \mathrm{yr}$, women $\geq 75 \mathrm{yr}$ ); blood urea nitrogen $\geq 21 \mathrm{mg} / \mathrm{dL}$ or presence of dehydration; $\mathrm{SpO}_{2} \leq 90 \%\left(\mathrm{PaO}_{2} \leq 60\right.$ Torr $)$; impaired consciousness (confusion); and low blood pressure (systolic blood pressure $\leq 90 \mathrm{mmHg}$ ). The number of positive items reflects the severity of the pneumonia, which influences the patient's prognosis. Thus, they recommend that patients with scores of $\geq 3$ receive treatment in hospital. The Japanese CAP guidelines also emphasize the importance of using rapid diagnostic tests to differentiate between atypical and bacterial pneumonia to allow selection of an appropriate antibiotic. In the present case, our patient's score was 1 (age: $80 \mathrm{yr}$ old) and the results of both a Gram stain of sputum and the urine pneumococcal rapid antigen test suggested bacterial pneumonia, specifically pneumonia caused by $S$. pneumoniae. According to the guidelines, either treatment at home with oral medication or in hospital with intravenous antibiotics is appropriate. However, sudden unpredictable deterioration due to flourishing of BLNAR $H$. influenzae occurred after substitution of ampicillin for meropenem. High dose ampicillin did not prevent this patient from deteriorating because of strong resistance of BLNAR $H$. influenzae to ampicillin. It is noteworthy that the BLNAR $H$. influenzae isolated from this patient was sensitive to macrolides and fluoroquinolones. Combined macrolide- $\beta$-lactam or fluoroquinolone- $\beta$-lactam therapy might have altered the clinical course of the present case.
We also provided this patient with nutritional support. Initially, we started enteral feeding with Oxepa (Abott), a unique product that contains eicosapentaenoic (EPA) and gamma-linolenic acids (GLA). Acute respiratory distress syndrome patients fed an enteral diet containing EPA and GLA and large amounts of various antioxidants (Oxepa) have significantly reduced pulmonary inflammation, increased oxygenation, and improved clinical outcomes than patients not fed this product [15]. Feeding of acute respiratory distress syndrome patients with Oxepa results not only in a reduction in neutrophils in bronchoalveolar lavage fluid (BALF) and in alveolar membrane protein permeability, but also in a decrease in amounts of interleukin-8 and leukotriene B4 in BALF [16]. Thus, early feeding with Oxepa may have contributed to the clinical improvement of this patient.

In conclusion, we here describe a rare case of an elderly patient with severe bacterial pneumonia, initially due to $S$. pneumoniae, that developed into coinfection with BLNAR $H$. influenzae. Although we conformed to the guidelines provided by the JRS for the management of CAP in adults, we were unable to prevent worsening of this patient's bacterial pneumonia. Prudent decisions are necessary when choosing the initial antibiotics for CAP patients.

\section{Acknowledgement}

This work was done at Social Insurance Gunma Chuo General Hospital, Maebashi, Gunma, Japan.

\section{Conflict of Interest}

No conflict of interest.

\section{Grant Support}

No funding.

\section{References}

1. Ohkusu K, Nakamura A, Sawada K. Antibiotic resistance among recent clinical isolates of Haemophilus influenzae in Japanese children. Diagn Microbiol Infect Dis. 2000;36(4):249-254.

2. Seki H, Kasahara Y, Ohta K, Saikawa Y, Sumita R, Yachie A, Fujita S, et al. Increasing prevalence of ampicillin- resistant, non-beta-lactamase-producing strains of Haemophilus influenzae in children in Japan. Chemotherapy. 1999;45(1):15-21.

3. Hasegawa K, Kobayashi R, Takada E, Ono A, Chiba N, Morozumi M, Iwata $\mathrm{S}$, et al. High prevalence of type $\mathrm{b}$ 
beta-lactamase-non-producing ampicillin-resistant Haemophilus influenzae in meningitis: the situation in Japan where Hib vaccine has not been introduced. J Antimicrob Chemother. 2006;57(6):1077-1082.

4. Bartlett JG, Breiman RF, Mandell LA, File TM, Jr. Community-acquired pneumonia in adults: guidelines for management. The Infectious Diseases Society of America. Clin Infect Dis. 1998;26(4):811-838.

5. Lim WS, Macfarlane JT, Boswell TC, Harrison TG, Rose D, Leinonen M, Saikku P. Study of community acquired pneumonia aetiology (SCAPA) in adults admitted to hospital: implications for management guidelines. Thorax. 2001;56(4):296-301.

6. Miyashita N, Fukano H, Mouri K, Fukuda M, Yoshida K, Kobashi Y, Niki Y, et al. Community-acquired pneumonia in Japan: a prospective ambulatory and hospitalized patient study. J Med Microbiol. 2005;54(Pt 4):395400.

7. Appelbaum PC. Antimicrobial resistance in Streptococcus pneumoniae: an overview. Clin Infect Dis. 1992;15(1):77-83.

8. Dowson CG, Hutchison A, Spratt BG. Extensive remodelling of the transpeptidase domain of penicillinbinding protein $2 \mathrm{~B}$ of a penicillin-resistant South African isolate of Streptococcus pneumoniae. Mol Microbiol. 1989;3(1):95-102.

9. Yanagihara K, Otsu Y, Ohno H, Higashiyama Y, Miyazaki Y, Hirakata Y, Tomono K, et al. Clinical characteristics of pneumonia caused by penicillin resistant and sensitive Streptococcus pneumoniae in Japan. Intern Med. 2004;43(11):1029-1033.

10. Tristram S, Jacobs MR, Appelbaum PC. Antimicrobial resistance in Haemophilus influenzae. Clin Microbiol
Rev. 2007;20(2):368-389.

11. Fluit AC, Florijn A, Verhoef J, Milatovic D. Susceptibility of European beta-lactamase-positive and -negative Haemophilus influenzae isolates from the periods 1997/1998 and 2002/2003. J Antimicrob Chemother. 2005;56(1):133-138.

12. Mandell LA, Bartlett JG, Dowell SF, File TM, Jr., Musher DM, Whitney C. Update of practice guidelines for the management of community-acquired pneumonia in immunocompetent adults. Clin Infect Dis. 2003;37(11):1405-1433.

13. Niederman MS, Mandell LA, Anzueto A, Bass JB, Broughton WA, Campbell GD, Dean N, et al. Guidelines for the management of adults with community-acquired pneumonia. Diagnosis, assessment of severity, antimicrobial therapy, and prevention. Am J Respir Crit Care Med. 2001;163(7):1730-1754.

14. Miyashita N, Matsushima T, Oka M, Japanese Respiratory S. The JRS guidelines for the management of community-acquired pneumonia in adults: an update and new recommendations. Intern Med. 2006;45(7):419-428.

15. Gadek JE, DeMichele SJ, Karlstad MD, Pacht ER, Donahoe M, Albertson TE, Van Hoozen C, et al. Effect of enteral feeding with eicosapentaenoic acid, gamma-linolenic acid, and antioxidants in patients with acute respiratory distress syndrome. Enteral Nutrition in ARDS Study Group. Crit Care Med. 1999;27(8):1409-1420.

16. Pacht ER, DeMichele SJ, Nelson JL, Hart J, Wennberg AK, Gadek JE. Enteral nutrition with eicosapentaenoic acid, gamma-linolenic acid, and antioxidants reduces alveolar inflammatory mediators and protein influx in patients with acute respiratory distress syndrome. Crit Care Med. 2003;31(2):491-500. 